TAIWANESE JOURNAL OF MATHEMATICS

Vol. 14, No. 3B, pp. 1023-1046, June 2010

This paper is available online at http://www.tjm.nsysu.edu.tw/

\title{
NONEXPANSIVE RETRACTIONS ONTO CLOSED CONVEX CONES IN BANACH SPACES
}

\author{
Takashi Honda, Wataru Takahashi and Jen-Chih Yao* \\ Dedicated to the Memory of Professor Sen-Yen Shaw
}

\begin{abstract}
Let $E$ be a smooth, strictly convex and reflexive Banach space, let $C^{*}$ be a closed convex subset of the dual space $E^{*}$ of $E$ and let $\Pi_{C^{*}}$ be the generalized projection of $E^{*}$ onto $C^{*}$. Then the mapping $R_{C^{*}}$ defined by $R_{C^{*}}=J^{-1} \Pi_{C^{*}} J$ is a sunny generalized nonexpansive retraction of $E$ onto $J^{-1} C^{*}$, where $J$ is the normalized duality mapping on $E$. In this paper, we first prove that if $K$ is a closed convex cone in $E$ and $P$ is the nonexpansive retaction of $E$ onto $K$, then $P$ a sunny generalized nonexpansive retraction of $E$ onto $K$. Using this result, we obtain an equivalent condition for a closed half-space of $E$ to be a nonexpansive retract of $E$.
\end{abstract}

\section{INTRODUCTION}

Let $E$ be a smooth, Banach space and let $E^{*}$ be the dual space of $E$. The function $\phi: E \times E \rightarrow \mathbb{R}$ is defined by

$$
\phi(x, y)=\|x\|^{2}-2\langle x, J y\rangle+\|y\|^{2}
$$

for each $x, y \in E$, where $J$ is the normalized duality mapping from $E$ into $E^{*}$. Let $C$ be a nonempty closed convex subset of $E$ and let $T$ be a mapping from $C$ into itself. Then, $T$ is called generalized nonexpansive if the set $F(T)$ of fixed points of $T$ is nonempty and

$$
\phi(T x, y) \leq \phi(x, y)
$$

for all $x \in C$ and $y \in F(T)$; see Ibaraki and Takahashi [22]. Such nonlinear operators are connected with the resolvents of maximal monotone operators in Banach

Received November 5, 2009.

2000 Mathematics Subject Classification: Primary 47H09; Secondary 47H10, 60 G05.

Key words and phrases: Relatively nonexpansive mapping, Generalized nonexpansive mapping, Generalized projection, Sunny generalized nonexpansive retraction, Fixed point, Conditional expectation. *Corresponding author. 
spaces. When $E$ is a smooth, strictly convex and reflexive Banach space and $C$ is a nonempty closed convex subset of $E$, Alber [1] also defined a nonlinear projection $\Pi_{C}$ of $E$ onto $C$ called the generalized projection. Motivated by Alber [1] and Ibaraki and Takahashi [22], Kohsaka and Takahashi [29] proved the following result: Let $E$ be a smooth, strictly convex and reflexive Banach space, let $C^{*}$ be a nonempty closed convex subset of $E^{*}$ and let $\Pi_{C^{*}}$ be the generalized projection of $E^{*}$ onto $C^{*}$. Then the mapping $R$ defined by $R=J^{-1} \Pi_{C^{*}} J$ is a sunny generalized nonexpansive retraction of $E$ onto $J^{-1} C^{*}$.

When $E$ is a Hilbert space and $C$ is a closed convex subset of $E$, the metric projection (the nearest point projection) of $E$ onto $C$, a sunny nonexpansive retraction of $E$ onto $C$, the generalized projection of $E$ onto $C$ and a sunny generalized nonexpansive retraction of $E$ onto $C$ are all same; see [36]. However, it is known [32] that if the metric projections are nonexpansive whenever they exist for closed convex subsets $C$ of a Banach space $E$ with $\operatorname{dim}(E) \geq 3$, then $E$ must be a Hilbert space. Moreover, it is also known [34] that if every closed convex subset of a Banach space $E$ with $\operatorname{dim}(E) \geq 3$ is a nonexpansive retract of $E$, then $E$ is necessarily a Hilbert space; see also [30].

Motivated by Ibaraki and Takahashi [22], Honda and Takahashi [18, 19] obtained the relation between nonexpansive retractions and sunny generalized nonexpansive retractions in a Banach space when their retracts of $E$ are closed linear subspaces.

In this paper, we study the relation between nonexpansive retractions and sunny generalized nonexpansive retractions in a Banach space when their retarcts of $E$ are closed convex cones. Furthermore, we obtain an equivalent condition for a closed half space of a Banach space $E$ to be a nonexpansive retract of $E$.

\section{PReliminaries}

Throughout this paper, $E$ is a real Banach space with the dual $E^{*}$. For any subset $A$ of $E, \bar{A}$ denotes the closure of $A$ with respect to the norm topology, Int $A$ denotes the set of interior points of $A$ with respect to the norm topology and $\partial A$ denotes the set of boundary points of $A$ with respect to the norm topology. We denote by $\mathbb{N}$ and $\mathbb{R}$ the sets of all positive integers and all real numbers, respectively. We also denote by $\left\langle x, x^{*}\right\rangle$ the dual pair of $x \in E$ and $x^{*} \in E^{*}$. A Banach space $E$ is said to be strictly convex if $\|x+y\|<2$ for $x, y \in E$ with $\|x\| \leq 1,\|y\| \leq 1$ and $x \neq y$. A Banach space $E$ is said to be smooth provided

$$
\lim _{t \rightarrow 0} \frac{\|x+t y\|-\|x\|}{t}
$$

exists for each $x, y \in E$ with $\|x\|=\|y\|=1$. Let $E$ be a Banach space. With 
each $x \in E$, we associate the set

$$
J(x)=\left\{x^{*} \in E^{*}:\left\langle x, x^{*}\right\rangle=\|x\|^{2}=\left\|x^{*}\right\|^{2}\right\} .
$$

The multivalued operator $J: E \rightarrow E^{*}$ is called the normalized duality mapping of $E$. From the Hahn-Banach theorem, $J x \neq \emptyset$ for each $x \in E$. We know that $E$ is smooth if and only if $J$ is single-valued. If $E$ is strictly convex, then $J$ is one-toone, i.e., $x \neq y \Rightarrow J(x) \cap J(y)=\emptyset$. If $E$ is reflexive, then $J$ is a mapping of $E$ onto $E^{*}$. So, if $E$ is reflexive, strictly convex and smooth, then $J$ is single-valued, one-to-one and onto. In this case, the normalized duality mapping $J_{*}$ from $E^{*}$ into $E$ is the inverse of $J$, that is, $J_{*}=J^{-1}$; see [36] for more details. Let $E$ be a smooth Banach space and let $J$ be the normalized duality mapping of $E$. We define the function $\phi: E \times E \rightarrow \mathbb{R}$ by

$$
\phi(x, y)=\|x\|^{2}-2\langle x, J y\rangle+\|y\|^{2}
$$

for all $x, y \in E$. We also define the function $\phi_{*}: E^{*} \times E^{*} \rightarrow \mathbb{R}$ by

$$
\phi_{*}\left(x^{*}, y^{*}\right)=\left\|x^{*}\right\|^{2}-2\left\langle x^{*}, J^{-1} y^{*}\right\rangle+\left\|y^{*}\right\|^{2}
$$

for all $x^{*}, y^{*} \in E^{*}$. It is easy to see that $(\|x\|-\|y\|)^{2} \leq \phi(x, y) \leq(\|x\|+\|y\|)^{2}$ for all $x, y \in E$. Thus, in particular, $\phi(x, y) \geq 0$ for all $x, y \in E$. We also know the following:

$$
\phi(x, y)=\phi(x, z)+\phi(z, y)+2\langle x-z, J z-J y\rangle
$$

for all $x, y, z \in E$. Further, we have

$$
2\langle x-y, J z-J w\rangle=\phi(x, w)+\phi(y, z)-\phi(x, z)-\phi(y, w)
$$

for all $x, y, z, w \in E$. It is easy to see that

$$
\phi(x, y)=\phi_{*}(J y, J x)
$$

for all $x, y \in E$. If $E$ is additionally assumed to be strictly convex, then

$$
\phi(x, y)=0 \Leftrightarrow x=y .
$$

The following lemma is well-known.

Lemma 2.1. ([28]). Let $E$ be a smooth and uniformly convex Banach space and let $\left\{x_{n}\right\}$ and $\left\{y_{n}\right\}$ be sequences in $E$ such that either $\left\{x_{n}\right\}$ or $\left\{y_{n}\right\}$ is bounded. If $\lim _{n \rightarrow \infty} \phi\left(x_{n}, y_{n}\right)=0$, then $\lim _{n \rightarrow \infty}\left\|x_{n}-y_{n}\right\|=0$. 
Let $C$ be a nonempty closed convex subset of a smooth, strictly convex and reflexive Banach space $E$. For an arbitrary point $x$ of $E$, the set

$$
\left\{z \in C: \phi(z, x)=\min _{y \in C} \phi(y, x)\right\}
$$

is always nonempty and a singleton. Let us define the mapping $\Pi_{C}$ of $E$ onto $C$ by $z=\Pi_{C} x$ for every $x \in E$, i.e.,

$$
\phi\left(\Pi_{C} x, x\right)=\min _{y \in C} \phi(y, x)
$$

for every $x \in E$. Such $\Pi_{C}$ is called the generalized projection of $E$ onto $C$; see Alber [1]. The following lemma is due to Alber [1] and Kamimura and Takahashi [28].

Lemma 2.2. ([1,28]). Let $C$ be a nonempty closed convex subset of a smooth, strictly convex and reflexive Banach space $E$ and let $(x, z) \in E \times C$. Then, the following hold:

(a) $z=\Pi_{C} x$ if and only if $\langle y-z, J x-J z\rangle \leq 0$ for all $y \in C$;

(b) $\phi\left(z, \Pi_{C} x\right)+\phi\left(\Pi_{C} x, x\right) \leq \phi(z, x)$.

Let $D$ be a nonempty closed convex subset of a smooth Banach space $E$, let $T$ be a mapping from $D$ into itself and let $F(T)$ be the set of fixed points of $T$. Then, $T$ is said to be generalized nonexpansive [22] if $F(T)$ is nonempty and $\phi(T x, u) \leq \phi(x, u)$ for all $x \in D$ and $u \in F(T)$. Let $C$ be a nonempty subset of $E$ and let $R$ be a mapping from $E$ onto $C$. Then $R$ is said to be a retraction, or a projection if $R x=x$ for all $x \in C$. It is known that if a mapping $P$ of $E$ into $E$ satisfies $P^{2}=P$, then $P$ is a projection of $E$ onto $\{P x: x \in E\}$. A mapping $T: E \rightarrow E$ with $F(T) \neq \emptyset$ is a retraction if and only if $F(T)=r(T)$, where $r(T)$ is the range of $T$. When a mapping $T$ is a retraction, the subset $r(T)$ is said to be a retract. The mapping $R$ is also said to be sunny if $R(R x+t(x-R x))=R x$ whenever $x \in E$ and $t \geq 0$. A nonempty subset $C$ of a smooth Banach space $E$ is said to be a generalized nonexpansive retract (resp. sunny generalized nonexpansive retract) of $E$ if there exists a generalized nonexpansive retraction (resp. sunny generalized nonexpansive retraction) $R$ from $E$ onto $C$. The following lemmas were proved by Ibaraki and Takahashi [22].

Lemma 2.3. ([22]). Let $C$ be a nonempty closed subset of a smooth, strictly convex and reflexisve Banach space $E$ and let $R$ be a retraction from $E$ onto $C$. Then, the following are equivalent:

(a) $R$ is sunny and generalized nonexpansive;

(b) $\langle x-R x, J y-J R x\rangle \leq 0$ for all $(x, y) \in E \times C$. 
Lemma 2.4. ([22]). Let $C$ be a nonempty closed sunny and generalized nonexpansive retract of a smooth and strictly convex Banach space E. Then, the sunny generalized nonexpansive retraction from $E$ onto $C$ is uniquely determined.

Lemma 2.5. ([22]). Let $C$ be a nonempty closed subset of a smooth and strictly convex Banach space $E$ such that there exists a sunny generalized nonexpansive retraction $R$ from $E$ onto $C$ and let $(x, z) \in E \times C$. Then, the following hold:

(a) $z=R x$ if and only if $\langle x-z, J y-J z\rangle \leq 0$ for all $y \in C$;

(b) $\phi(R x, z)+\phi(x, R x) \leq \phi(x, z)$.

Let $C$ be a nonempty closed convex subset of a smooth, strictly convex and reflexive Banach space $E$. For an arbitrary point $x$ of $E$, the set

$$
\left\{z \in C:\|z-x\|=\min _{y \in C}\|y-x\|\right\}
$$

is always nonempty and a singleton. Let us define the mapping $P_{C}$ of $E$ onto $C$ by $z=P_{C} x$ for every $x \in E$, i.e.,

$$
\left\|P_{C} x-x\right\|=\min _{y \in C}\|y-x\|
$$

for every $x \in E$. Such $P_{C}$ is called the metric projection of $E$ onto $C$; see [36]. The following lemma is in [36].

Lemma 2.6. ([36]). Let $C$ be a nonempty closed convex subset of a smooth, strictly convex and reflexive Banach space $E$ and let $(x, z) \in E \times C$. Then, $z=P_{C} x$ if and only if $\langle y-z, J(x-z)\rangle \leq 0$ for all $y \in C$.

An operator $A: E \rightarrow 2^{E^{*}}$ with domain $D(A)=\{x \in E: A x \neq \emptyset\}$ and range $r(A)=\cup\{A x: x \in D(A)\}$ is said to be monotone if $\left\langle x-y, x^{*}-y^{*}\right\rangle \geq 0$ for any $\left(x, x^{*}\right),\left(y, y^{*}\right) \in A$. The operator $A$ is said to be strictly monotone if $\left\langle x-y, x^{*}-y^{*}\right\rangle>0$ for any $x, y \in E, x^{*} \in A x, y^{*} \in A y$. A monotone operator $A$ is said to be maximal if its graph $G(A)=\left\{\left(x, x^{*}\right): x^{*} \in A x\right\}$ is not properly contained in the graph of any other monotone operator. If $A$ is maximal monotone, then the set $A^{-1} 0=\{u \in E: 0 \in A u\}$ is closed and convex (see [37] for more details). Let $J$ be the normalized duality mapping from $E$ into $E^{*}$. Then, $J$ is monotone. If $E$ is strictly convex, then $J$ is one to one and strictly monotone. The following theorem is well-known; for instance, see [36].

Theorem 2.1. Let $E$ be a reflexive, strictly convex and smooth Banach space and let $A: E \rightarrow 2^{E^{*}}$ be a monotone operator. Then $A$ is maximal if and only if $r(J+r A)=E^{*}$ for all $r>0$. Further, if $r(J+A)=E^{*}$, then $r(J+r A)=E^{*}$ for all $r>0$. 


\section{Nonexpansive Retractions onto Closed Convex Cones}

In this section, we discuss some relations between a nonexpansive retraction onto a closed convex cone and sunny generalized nonexpansive retraction. We start with two theorems proved by Kohsaka and Takahashi [29].

Theorem 3.1. ([29]). Let $E$ be a smooth, strictly convex and reflexive Banach space, let $C^{*}$ be a nonempty closed convex subset of $E^{*}$ and let $\Pi_{C^{*}}$ be the generalized projection of $E^{*}$ onto $C^{*}$. Then the mapping $R$ defined by $R=$ $J^{-1} \Pi_{C^{*}} J$ is a sunny generalized nonexpansive retraction of $E$ onto $J^{-1} C^{*}$.

Theorem 3.2. ([29]). Let $E$ be a smooth, reflexive and strictly convex Banach space and let $D$ be a nonempty subset of $E$. Then, the following conditions are equivalent.

(1) $D$ is a sunny generalized nonexpansive retract of $E$;

(2) $D$ is a generalized nonexpansive retract of $E$;

(3) JD is closed and convex.

In this case, $D$ is closed.

From these theorems, we can represent sunny generalized nonexpansive retraction by using generalized projections. Let $E$ be a reflexive, strictly convex and smooth Banach space and let $J$ be the normalized duality mapping from $E$ onto $E^{*}$. Let $C^{*}$ be a closed convex subset of the dual space $E^{*}$ of $E$. Then, the sunny generalized nonexpansive retraction $R_{C^{*}}$ with respect to $C^{*}$ is defined as follows:

$$
R_{C^{*}}:=J^{-1} \Pi_{C^{*}} J
$$

where $\Pi_{C^{*}}$ is the generalized projection from $E^{*}$ onto $C^{*}$.

Let $Y$ be a nonempty subset of a Banach space $E$ and let $Y^{*}$ be a nonempty subset of the dual space $E^{*}$. Then, we define the annihilator $Y_{\perp}^{*}$ of $Y^{*}$ and the annihilator $Y^{\perp}$ of $Y$ as follows:

$$
Y_{\perp}^{*}=\left\{x \in E: f(x)=0 \text { for all } f \in Y^{*}\right\}
$$

and

$$
Y^{\perp}=\left\{f \in E^{*}: f(x)=0 \text { for all } x \in Y\right\} .
$$

In a reflexive Banach space, both concepts coincide with each other.

Let $E$ be a Banach space and let $C$ be a nonempty closed convex subset of $E$. Then, a mapping $T$ of $C$ into itself is nonexpansive if $\|T x-T y\| \leq\|x-y\|$ for all $x, y \in C$. A mapping $T$ of $C$ into itself with $F(T) \neq \emptyset$ is said to be quasi-nonexpansive if $\|T x-m\| \leq\|x-m\|$ for all $m \in F(T)$ and $x \in C$. It is clear that any nonexpansive mapping with fixed points is quasi-nonexpansive.

Motivated by previous theorems, the authors obtained following theorems. 
Theorem 3.3. ([3,18]). Let $E$ be a reflexive, strictly convex and smooth Banach space and let $I$ be the identity operator of $E$ into itself. Let $Y^{*}$ be a closed linear subspace of the dual space $E^{*}$ and let $R_{Y^{*}}$ be the sunny generalized nonexpansive retraction with respect to $Y^{*}$. Then, the mapping $I-R_{Y^{*}}$ is the metric projection of $E$ onto $Y_{\perp}^{*}$. Conversely, let $Y$ be a closed linear subspace of $E$ and let $P_{Y}$ be the metric projection of $E$ onto $Y$. Then, the mapping $I-P_{Y}$ is the generalized conditional expectation $R_{Y^{\perp}}$ with respect to $Y^{\perp}$, i.e., $I-P_{Y}=R_{Y^{\perp}}$.

Theorem 3.4. ([19]). Let $E$ be a strictly convex, reflexive and smooth Banach space and let $Y^{*}$ be a closed linear subspace of the dual space $E^{*}$ of $E$. If the sunny generalized nonexpansive retraction $R_{Y^{*}}$ is a quasi-nonexpansive projection of $E$ onto $J^{-1} Y^{*}$, then it is a norm one linear projection and $J^{-1} Y^{*}$ is a closed linear subspace in E. Conversely, any norm one linear projection is a quasinonexpansive sunny generalized nonexpansive retraction with respect some closed linear subspace in $E^{*}$.

We shall generalize these theorems and obtain a nonlinear retraction which is both "nonexpansive" and "sunny generalized nonexpansive".

A subset $K$ of a Banach space is called a cone if it satisfies that $\lambda x \in K$ when $x \in K$ and $\lambda \geq 0$. Any cone contains the origin. When a cone contains a non-zero element, we call it nontrivial.

Theorem 3.5. Let $E$ be a reflexive and smooth Banach space and let $K$ be a closed convex cone in $E$ If $T: K \rightarrow K$ is a quasi-nonexpansive mapping such that $F(T)$ is a cone, then $T$ is generalized nonexpansive.

Proof. We first show that for any $x \in K$ and $m \in F(T)$,

$$
\langle x-T x, J m\rangle \leq 0,
$$

where $J$ is the normalized duality mapping of $E$.

For the case of $m=0$, it is obvious that $\langle x-T x, J m\rangle=0$.

Fix $x \in K \backslash F(T)$ and $m \in F(T)$ such that $m \neq 0$. We have that for all $\alpha \in \mathbb{R}$ with $\alpha>0$,

$$
x \in F(T) \Leftrightarrow \alpha x \in F(T) .
$$

So, we have that $\frac{x}{k}-m \neq 0$ for any $k>0$. We have from the Hahn-Banach theorem that there exists $\xi_{k} \in E^{*}$ such that $\left\langle\frac{x}{k}-m, \xi_{k}\right\rangle=\left\|\frac{x}{k}-m\right\|$ and $\left\|\xi_{k}\right\|=1$. Then, we have that

$$
\left\langle\frac{T x}{k}-m, \xi_{k}\right\rangle \leq\left\|\frac{T x}{k}-m\right\|=\frac{1}{k}\|T x-k m\|
$$




$$
\begin{aligned}
& \leq \frac{1}{k}\|x-k m\|=\left\|\frac{x}{k}-m\right\| \\
& =\left\langle\frac{x}{k}-m, \xi_{k}\right\rangle .
\end{aligned}
$$

So, we have $\left\langle\frac{x}{k}-\frac{T x}{k}, \xi_{k}\right\rangle \geq 0$ and hence

$$
\left\langle x-T x, \xi_{k}\right\rangle \geq 0 .
$$

Take a positive sequence $\left\{k_{n}\right\}$ with $k_{n} \rightarrow \infty$. Put $x_{n}=\frac{x}{k_{n}}-m$ and $\xi_{n}=\xi_{k_{n}}$. Then, we have $\frac{x}{k_{n}}-m \rightarrow-m$. Since $E$ is a reflexive Banach space and $\left\{\xi_{n}\right\}$ is bounded, there exists a subsequence $\left\{\xi_{n_{i}}\right\}$ of $\left\{\xi_{n}\right\}$ converging to some $\xi \in E^{*}$ in weak topology.

We may show that $\xi$ satisfies $\langle m,-\xi\rangle=\|m\|$ and $\|\xi\|=1$. Since the norm of $E^{*}$ is lower semicontinuous in the weak topology, we have

$$
\|\xi\| \leq \liminf _{i \rightarrow \infty}\left\|\xi_{n_{i}}\right\|=1
$$

On the other hand, we have that

$$
\begin{aligned}
\left|\langle-m, \xi\rangle-\left\|x_{n_{i}}\right\|\right| & =\left|\langle-m, \xi\rangle-\left\langle x_{n_{i}}, \xi_{n_{i}}\right\rangle\right| \\
& \leq\left|\left\langle-m, \xi-\xi_{n_{i}}\right\rangle\right|+\left|\left\langle-m-x_{n_{i}}, \xi_{n_{i}}\right\rangle\right| .
\end{aligned}
$$

Since $\left\langle-m, \xi-\xi_{n_{i}}\right\rangle \rightarrow 0$ and $\left\langle-m-x_{n_{i}}, \xi_{n_{i}}\right\rangle \rightarrow 0$, we have

$$
\left\|x_{n_{i}}\right\| \rightarrow-\langle m, \xi\rangle=\langle m,-\xi\rangle \text {. }
$$

Since $\left\|x_{n_{i}}\right\| \rightarrow\|m\|$, we have $\langle m,-\xi\rangle=\|m\|$. So we have

$$
\|m\|=\langle m,-\xi\rangle \leq\|m\|\|\xi\|
$$

and hence $\|\xi\| \geq 1$. Therefore, we have $\|\xi\|=1$ and $\langle m,-\xi\rangle=\|m\|$. Then, without loss of generality, there exists a positive sequence $\left\{k_{n}\right\}$ such that

$$
\begin{gathered}
k_{n} \rightarrow \infty, \\
\frac{x}{k_{n}}-m \rightarrow-m
\end{gathered}
$$

and

$$
\xi_{k_{n}} \rightarrow \xi
$$

in weak topology, where $\xi$ is an element of $E^{*}$ such that $\langle m,-\xi\rangle=\|m\|$ and $\|\xi\|=1$.

Putting $\xi_{0}=-\xi$, we have $\left\langle m, \xi_{0}\right\rangle=\|m\|,\left\|\xi_{0}\right\|=1$ and

$$
\left\langle x-T x, \xi_{0}\right\rangle \leq 0 .
$$


Since $E^{*}$ is smooth and

$$
\|\| m\left\|\xi_{0}\right\|^{2}=\|m\|^{2}=\|m\|\left\langle m, \xi_{0}\right\rangle=\left\langle m,\|m\| \xi_{0}\right\rangle
$$

we know that $\|m\| \xi_{0}=J m$, where $J$ is the normalized duality mapping on $E$. Then for any $x \in K \backslash F(T)$ and $m \in F(T) \backslash\{0\}$, we have $\|m\|\left\langle x-T x, \xi_{0}\right\rangle \leq 0$ and hence

$$
\langle x-T x, J m\rangle \leq 0 .
$$

We also have for $x \in F(T)$ and $m \in F(T)$ with $m \neq 0,\langle x-T x, J m\rangle=0$.

So, the inequality (3.1) holds for any $x \in K$ and $m \in F(T)$. This implies that for any $x \in K$ and $m \in F(T)$,

$$
\langle x, J m\rangle \leq\langle T x, J m\rangle .
$$

Since $T$ is quasi-nonexpansive and $0 \in F(T)$, we have $\|T x\| \leq\|x\|$. Then for any $x \in E$ and $m \in K$, we have $\|T x\|^{2}-2\langle T x, J m\rangle+\|m\|^{2} \leq\|x\|^{2}-2\langle x, J m\rangle+\|m\|^{2}$ and hence

$$
\phi(T x, m) \leq \phi(x, m) .
$$

This means that $T$ is a generalized nonexpansive mapping.

From this theorem, we obtain following corollaries.

Corollary 3.1. Let $E$ be a smooth and reflexive Banach space and let $T: E \rightarrow$ $E$ be a norm one linear operator. Then, $T$ is generalized nonexpansive.

Corollary 3.2. Let $E$ be a strictly convex, smooth and reflexive Banach space and let $K$ be a cone in $E$. If $K$ is a nonexpansive retract of $E$, then $K$ is a closed convex cone in $E, K$ is a sunny generalized nonexpansive retract and $J K$ is a closed convex cone in $E^{*}$.

Proof. Since $K$ is a nonexpansive retract of $E$, there exists a nonexpansive retraction $T$ with $T(E)=F(T)=K$. So, from [24], $F(T)=K$ must be closed and convex. From Theorem 3.5, we also know that $T$ is a generalized nonexpansive retraction of $E$ onto $K$. From Theorem 3.2, $K$ is a sunny generalized nonexpansive retract and $J K$ is a closed convex subset in $E^{*}$. Since for any $x \in E$ and $\alpha \in \mathbb{R}$ we have $J(\alpha x)=\alpha J x$ from [36], $J K$ is a cone.

We shall extend Theorem 3.3; see also Alber [2], Hudzik, Wang and Sha [21]. First we shall introduce two new nonlinear operators. We call a mapping $T: E \rightarrow E$ a firmly generalized nonexpansive type [23], if it satisfies

$$
\phi(T x, T y)+\phi(T y, T x)+\phi(x, T x)+\phi(y, T y) \leq \phi(x, T y)+\phi(y, T x)
$$


for all $x, y \in E$. We call a mapping $S: E \rightarrow E$ a firmly metric operator [38], if it satisfies

$$
\begin{aligned}
& \phi(x-S x, y-S y)+\phi(y-S y, x-S x) \\
\leq & \phi(x, y-S y)+\phi(y, x-S x)-\phi(x, x-S x)-\phi(y, y-S y)
\end{aligned}
$$

for all $x, y \in E$.

Let $C$ be a nonempty subset of a Banach space $E$ and let $C^{*}$ be a nonempty subset of the dual space $E^{*}$. Then, we define the dual cone (or the polar cone) $C_{\circ}^{*}$ of $C^{*}$ and the dual cone (or the polar cone) $C^{\circ}$ of $C$ as follows:

$$
C_{\circ}^{*}=\left\{x \in E: f(x) \leq 0 \text { for all } f \in C^{*}\right\}
$$

and

$$
C^{\circ}=\left\{f \in E^{*}: f(x) \leq 0 \text { for all } x \in C\right\} .
$$

Both of them are closed convex cones. In a reflexive Banach space, both concepts coincide with each other.

Lemma 3.1. Let $E$ be a strictly convex, smooth and reflexive Banach space, let $C$ be a nonempty closed convex subset of $E$ and let $P_{C}$ be the metric projection of $E$ onto $C$. Then the mapping $T=I-P_{C}$ is a firmly generalized nonexpansive type of $E$ into $E$. In particular, if $0 \in C$, then $F(T)=P_{C}^{-1} 0=J^{-1} C^{\circ}$ and $J F(T)$ is a closed convex cone in $E^{*}$.

Proof. From Lemma 2.6, we have that for any $x, y \in E$,

$$
\left\langle J\left(x-P_{C} x\right), P_{C} x-P_{C} y\right\rangle \geq 0
$$

and

$$
\left\langle J\left(y-P_{C} y\right), P_{C} y-P_{C} x\right\rangle \geq 0 .
$$

Then we have

$$
\left\langle J\left(x-P_{C} x\right)-J\left(y-P_{C} y\right), P_{C} x-P_{C} y\right\rangle \geq 0 .
$$

Since $T x=x-P_{C} x$ and $T y=y-P_{C} y$, we obtain

$$
\langle J T x-J T y, x-T x-(y-T y)\rangle \geq 0 .
$$

From (2.2), we have

$$
\begin{aligned}
0 & \leq 2\langle J T x-J T y, x-T x-(y-T y)\rangle \\
& =2\langle J T x-J T y, x-y\rangle-2\langle J T x-J T y, T x-T y\rangle \\
& =\phi(x, T y)+\phi(y, T x)-\phi(x, T x)-\phi(y, T y)-\phi(T x, T y)-\phi(T y, T x) .
\end{aligned}
$$


So, $T$ is a firmly generalized nonexpansive type on $E$. If $0 \in C$, we have that

$$
\begin{aligned}
& P_{C} x=0 \\
\Leftrightarrow & x-P_{C} x=x \\
\Leftrightarrow & T x=x .
\end{aligned}
$$

Then $F(T)=P_{C}^{-1} 0$. From Lemma 2.6, we have

$$
\begin{aligned}
x \in F(T) & \Leftrightarrow x \in P_{C}^{-1} 0 \\
& \Leftrightarrow\langle J(x-0), 0-y\rangle \geq 0 \text { for any } y \in C \\
& \Leftrightarrow\langle J(x), y\rangle \leq 0 \text { for any } y \in C \\
& \Leftrightarrow J x \in C^{\circ} .
\end{aligned}
$$

Then we obtain

$$
J F(T)=C^{\circ}=\cap_{y \in C}\left\{x^{*} \in E^{*}:\left\langle x^{*}, y\right\rangle \leq 0\right\} .
$$

This is the intersection of closed convex cones of $E^{*}$. So, $J F(T)$ is a closed convex cone in $E^{*}$.

Lemma 3.2. Let $E$ be a strictly convex, smooth and reflexive Banach space and let $T: E \rightarrow E$ be a firmly generalized nonexpansive type such that $J F(T)$ is a nonempty closed convex subset in $E^{*}$ and $T(E)=F(T)$. Then, $T$ is a sunny generalized nonexpansive retraction of $E$ onto $F(T)$.

Proof. From (3.2), we know that a mapping $T: E \rightarrow E$ satisfies that

$$
\langle J T x-J T y, x-T x-(y-T y)\rangle \geq 0 .
$$

From assumptions of $T, F(T) \neq \emptyset$. For any $x \in E$ and $m \in F(T)$, we have

$$
\langle J T x-J m, x-T x\rangle \geq 0 .
$$

Since $T x \in F(T)$ and $J F(T)$ is closed and convex in $E^{*}$, we have, from Lemma 2.3, that $T$ is a sunny generalized nonexpansive retraction of $E$ onto $F(T)$.

Lemma 3.3. Let $E$ be a strictly convex, smooth and reflexive Banach space and let $T: E \rightarrow E$ be a firmly metric operator such that $F(T)$ is a nonempty closed convex subset in $E$ and $T(E)=F(T)$. Then $T$ is the metric projection of $E$ onto $F(T)$.

Proof. From (3.2), for any $x, y \in E$, we have

$$
\langle J(x-T x)-J(y-T y), T x-T y\rangle \geq 0 .
$$


Then for any $x \in E$ and $m \in F(T)$, we have

$$
\langle J(x-T x), T x-m\rangle \geq 0 .
$$

Since $F(T)$ is closed and convex and $T x \in F(T)$, the mapping $T$ is the metric projection of $E$ onto $F(T)$.

Theorem 3.6. Let $E$ be a strictly convex, smooth and reflexive Banach space. Let $K$ be a closed convex cone of $E$ and let $P_{K}$ be the metric projection of $E$ onto $K$. Then the mapping $T=I-P_{K}$ is a sunny generalized nonexpansive retraction of $E$ onto $J^{-1} K^{\circ}$, where $K^{\circ}$ is the dual cone of $K$.

Proof. From Lemma 2.6, we have

$$
\left\langle J\left(x-P_{K} x\right), P_{K} x-m\right\rangle \geq 0
$$

for any $x \in E$ and $m \in K$. From $0 \in K$, we have

$$
\left\langle J\left(x-P_{K} x\right), P_{K} x\right\rangle \geq 0 .
$$

From $2 P_{K} x \in K$, we also have

$$
\left\langle J\left(x-P_{K} x\right), P_{K} x\right\rangle \leq 0 .
$$

From these inequalities, we have

$$
\left\langle J\left(x-P_{K} x\right), P_{K} x\right\rangle=0 .
$$

So, we have, for any $x \in E$ and $m \in K$,

$$
\begin{aligned}
& \left\langle J\left(x-P_{K} x\right), P_{K} x-m\right\rangle \geq 0 \\
\Rightarrow & \left\langle J\left(x-P_{K} x\right), P_{K} x\right\rangle-\left\langle J\left(x-P_{K} x\right), m\right\rangle \geq 0 \\
\Rightarrow & \left\langle J\left(x-P_{K} x\right), m\right\rangle \leq 0 \\
\Rightarrow & \langle J T x, m\rangle \leq 0 .
\end{aligned}
$$

Then for any $x \in E$, we have $J T x \in K^{\circ}$. We have $T(E) \subset J^{-1} K^{\circ}$ and hence

$$
F(T) \subset T(E) \subset J^{-1} K^{\circ} .
$$

From Lemma 3.1, we have that $T$ is a firmly generalized nonexpansive type, $J F(T)$ is a closed convex cone in $E^{*}$ and $F(T)=J^{-1} K^{\circ}$. Since $T(E)=F(T)=$ $J^{-1} K^{\circ}$, from Lemma 3.2, $T$ is a sunny generalized nonexpansive retraction of $E$ onto $F(T)=J^{-1} K^{\circ}$. 
Theorem 3.7. Let $E$ be a strictly convex, smooth and reflexive Banach space. Let $K^{*}$ be a closed convex cone of $E^{*}$ and let $R_{K^{*}}=J^{-1} \Pi_{K^{*}} J$ be the sunny generalized nonexpansive retraction of $E$ onto $J^{-1} K^{*}$, where $\Pi_{K^{*}}$ is the generalized projection of $E^{*}$ onto $K^{*}$. Then, the mapping $T=I-R_{K^{*}}$ is the metric projection of $E$ onto the dual cone $K_{\circ}^{*}$ of $K^{*}$.

Proof. Since $0 \in J^{-1} K^{*}$, from Lemma 2.3, we have

$$
\begin{aligned}
x \in R_{K^{*}}^{-1} 0 & \Leftrightarrow R_{K^{*}} x=0 \\
& \Leftrightarrow\left\langle x-0, J 0-J J^{-1} m^{*}\right\rangle \geq 0 \text { for any } m^{*} \in K^{*} \\
& \Leftrightarrow\left\langle x, m^{*}\right\rangle \leq 0 \text { for any } m^{*} \in K^{*} \\
& \Leftrightarrow x \in K_{\circ}^{*} .
\end{aligned}
$$

Then we have that

$$
R_{K^{*}}^{-1} 0=K_{\circ}^{*} .
$$

From assumptions, we have

$$
\begin{aligned}
& R_{K^{*}} x=0 \\
\Leftrightarrow & x-R_{K^{*}} x=x \\
\Leftrightarrow & T x=x .
\end{aligned}
$$

Then we have that

$$
F(T)=R_{K^{*}}^{-1} 0 .
$$

So, we obtain that

$$
F(T)=K_{\circ}^{*} .
$$

Since a sunny generalized nonexpansive retraction is a firmly generalized nonexpansive type, $T$ is a firmly metric operator such that $F(T)=K_{\circ}^{*}$. To obtain the desired result, from Lemma 3.3, it is sufficient to show that $T(E) \subset F(T)=K_{\circ}^{*}$. From $0,2 R_{K^{*}} x \in J^{-1} K^{*}$ and Lemma 2.3, we have

$$
\left\langle x-R_{K^{*}} x, J R_{K^{*}} x\right\rangle=0 .
$$

So, we have for any $x \in E$ and $m^{*} \in K^{*},\left\langle x-R_{K^{*}} x, J R_{K^{*}} x-J J^{-1} m^{*}\right\rangle \geq 0$ and hence

$$
\left\langle x-R_{K^{*}} x, m^{*}\right\rangle \leq 0 .
$$

Then we have that for any $x \in E$ and $m^{*} \in K^{*}$,

$$
\left\langle T x, m^{*}\right\rangle \leq 0 .
$$

Then we obtain that $T x \in K_{\circ}^{*}$ for any $x \in E$. This implies $T(E) \subset K_{\circ}^{*}$. Therefore, $T=P_{K_{\circ}^{*}}$. This completes the proof. 
Remark 3.1. In a Hilbert space, Theorem 3.3 is called the Riesz decomposition and Theorems 3.6 and 3.7 are called the Moreau decomposition; see Hudzik, Wang and Sha [21].

From Corollary 3.2and Theorem 3.7, we have the following corollary.

Corollary 3.3. Let $E$ be a strictly convex, reflexive and smooth Banach space and let $K$ be a closed convex cone of $E$. If there exists a sunny nonexpansive retraction $R$ of $E$ onto $K$, then $I-R$ is the metric projection of $E$ onto $\{J K\}$, where $I$ is the identity mapping on $E$.

\section{Nonexpansive Retractions onto Closed Half-SPaces}

Let $E$ be a strictly convex, reflexive and smooth Banach space. Calvert [10] showed that a closed linear subspace $Y$ in $E$ is a 1-complemented subspace (i.e. the range of a norm one linear projection) if and only if $J Y$ is a closed linear subspace in $E^{*}$; see also [18]. Using our theorems in the preivious section, we can extend this result.

Let $E$ be a Banach space. A subset $V \subset E$ is called a linear manifold if it is of the form $V=\left\{x_{0}+g: g \in G\right\}$, where $x_{0}$ is some element of $E$ and $G$ is a linear subspace of $E$. We call a closed linear manifold $M$ a closed hyperplane if there exists no closed linear manifold $M_{1} \subset E$ such that $M \subset M_{1}$ and $M \neq M_{1} \neq E$. We know that $M$ is a closed hyperplane if and only if there exist a nonzero bounded linear functional $f \in E^{*}$ and $\alpha \in \mathbb{R}$ such that $M=\{x \in E: f(x)=\alpha\}$; see Singer [35]. A subset $H \subset E$ is called a closed half-space if it is of the form $H=\{x \in E: f(x) \leq \alpha\}$, where $f$ is a nonzero bounded linear functional $f \in E^{*}$ and $\alpha \in \mathbb{R}$. In particular, in this paper, a closed half-space means only the case $\alpha=0$.

Theorem 4.1. Let $E$ be a strictly convex, smooth and reflexive Banach space and let $H$ be a closed half-space of $E$ such that for some $z^{*} \in E^{*} \backslash\{0\}$

$$
H=\left\{x \in E:\left\langle x, z^{*}\right\rangle \leq 0\right\} .
$$

Then, $H$ is a nonexpansive retract of $E$ if and only if $J H$ is a closed half-space in $E^{*}$.

To prove this theorem, we need some definitions and lemmas. Let $E$ be a real Banach space. The definition of orthogonality that we use is that of Birkhoff [7] and James [25, 26, 27]; for $x, y \in E, x$ is said to be orthogonal to $y$, denoted by $x \perp y$, if

$$
\|x+\lambda y\| \geq\|x\|
$$


for all $\lambda \in \mathbb{R}$. $x$ is said to be acute to $y$ if (4.1) holds for all $\lambda \geq 0$. When $E$ is smooth, we know that

$$
x \text { is orthogonal to } y \Leftrightarrow\langle J x, y\rangle=0
$$

and

$$
x \text { is acute to } y \Leftrightarrow\langle J x, y\rangle \geq 0 ;
$$

see [36]. Let $F$ be a closed subset of $E$. A retraction $R$ of $E$ onto $F$ is orthogonal; see Bruck [9], if for each $x \in E$ and $m \in F, R x-m$ is acute to $x-R x$;

$$
\|(1-\lambda) R x+\lambda x-m\| \geq\|R x-m\|
$$

for all $\lambda \geq 0$.

Using this orthogonal retraction, we show a following lemma.

Lemma 4.1. Let $E$ be a strictly convex, smooth and reflexive Banach space and let $H$ be a closed half-space of $E$ such that for some $z^{*} \in E^{*} \backslash\{0\}$

$$
H=\left\{x \in E:\left\langle x, z^{*}\right\rangle \leq 0\right\} .
$$

Then, $H$ is a nonexpansive retract of $E$ if and only if $J H$ is a closed convex cone in $E^{*}$.

Proof. A closed half-space $H$ is a closed convex cone. If $H$ is a nonexpansive retract of $E$, from Corollary 3.2, $J H$ is a closed convex cone in $E^{*}$.

Conversely, if $J H$ is a closed convex cone in $E^{*}$, from Theorem 3.2, there exists the sunny generalized nonexpansive retraction $R_{J H}=J^{-1} \Pi_{J H} J$ of $E$ onto $H$, where $\Pi_{J H}$ is the generalized projection of $E^{*}$ onto $J H$. We shall show that $R_{J H}$ is nonexpansive. Since $R_{J H}$ is sunny, we have for any $x \in E$,

$$
R_{J H}\left(R_{J H} x+\lambda\left(x-R_{J H} x\right)\right)=R_{J H} x,
$$

for $\lambda \geq 0$. When $z \in E \backslash H=\left\{x \in E:\left\langle x, z^{*}\right\rangle>0\right\}$, we have that $R_{J H} z \in$ $\left\{x \in E:\left\langle x, z^{*}\right\rangle=0\right\}$. In fact, if $R_{J H} z \in\left\{x \in E:\left\langle x, z^{*}\right\rangle<0\right\}$, then $z-R_{J H} z \in\left\{x \in E:\left\langle x, z^{*}\right\rangle>0\right\}$. For a sufficiently small $\lambda>0$, we have

$$
R_{J H} z+\lambda\left(z-R_{J H} z\right) \in\left\{x \in E:\left\langle x, z^{*}\right\rangle<0\right\} \subset H .
$$

Then we have that

$$
R_{J H} z=R_{J H}\left(R_{J H} z+\lambda\left(z-R_{J H} z\right)\right)=R_{J H} z+\lambda\left(z-R_{J H} z\right)
$$

and hence $\lambda\left(z-R_{J H} z\right)=0$. From $\lambda>0$, we have $z-R_{J H} z=0$ and hence $z \in H=\left\{x \in E:\left\langle x, z^{*}\right\rangle \leq 0\right\}$. This contradicts to $z \in\left\{x \in E:\left\langle x, z^{*}\right\rangle>0\right\}$. 
So, for any $m \in H$ and $z \notin H$, we have

$$
m-R_{J H} z \in\left\{x \in E:\left\langle x, z^{*}\right\rangle \leq 0\right\}=H .
$$

Then $J\left(m-R_{J H} z\right) \in J H$. From Theorem 3.7, the mapping $P=I-R_{J H}$ is the metric projection of $E$ onto $(J H)_{\circ}$. Then we have, for any $m \in H$ and $z \notin H$,

$$
\begin{aligned}
& \left\langle J\left(m-R_{J H} z\right), P z\right\rangle \leq 0 \\
\Rightarrow & \left\langle J\left(m-R_{J H} z\right), z-R_{J H} z\right\rangle \leq 0 \\
\Rightarrow & \left\langle J\left(R_{J H} z-m\right), z-R_{J H} z\right\rangle \geq 0 .
\end{aligned}
$$

From this, we obtain that $R_{J H} z-m$ is acute to $z-R_{J H} z$. When $z \in H$, $z-R_{J H} z=0$ and $R_{J H} z-m$ is acute to $z-R_{J H} z$ obviously. This means that $R_{J H}$ is an orthogonal retraction of $E$ onto $H$. Since $R_{J H}$ is an orthogonal retraction of $E$ onto $H$, for any $x, y \in E$, we have

$$
\left\langle J\left(R_{J H} x-R_{J H} y\right), x-R_{J H} x\right\rangle \geq 0
$$

and

$$
\left\langle J\left(R_{J H} y-R_{J H} x\right), y-R_{J H} y\right\rangle \geq 0 .
$$

Then for any $x, y \in E$, we have

$$
\begin{aligned}
& \left\langle J\left(R_{J H} x-R_{J H} y\right), x-R_{J H} x\right\rangle-\left\langle J\left(R_{J H} x-R_{J H} y\right), y-R_{J H} y\right\rangle \geq 0 \\
\Rightarrow & \left\langle J\left(R_{J H} x-R_{J H} y\right), x-y-\left(R_{J H} x-R_{J H} y\right)\right\rangle \geq 0 \\
\Rightarrow & \left\langle J\left(R_{J H} x-R_{J H} y\right), x-y\right\rangle \geq\left\|R_{J H} x-R_{J H} y\right\|^{2} \\
\Rightarrow & \left\|R_{J H} x-R_{J H} y\right\| \cdot\|x-y\| \geq\left\|R_{J H} x-R_{J H} y\right\|^{2} \\
\Rightarrow & \|x-y\| \geq\left\|R_{J H} x-R_{J H} y\right\| .
\end{aligned}
$$

Then $R_{J H}$ is nonexpansive. So, $H$ is a nonexpansive retract of $E$.

Using an idea of Beauzamy [5] and Davis and Enflo [12], we obtain the following lemma.

Lemma 4.2. Let $E$ be a strictly convex, smooth and reflexive Banach space and let $H$ be a closed half-space of $E$ such that for some $z^{*} \in E^{*} \backslash\{0\}$

$$
H=\left\{x \in E:\left\langle x, z^{*}\right\rangle \leq 0\right\} .
$$

Let $M=\left\{x \in E:\left\langle x, z^{*}\right\rangle=0\right\}$. Then, $H$ is a nonexpansive retract of $E$ if and only if $J M$ is a closed linear subspace of $E^{*}$. 
Proof. Assume that $H$ is a nonexpansive retract of $E$. Then, from Corollary $3.2, J H$ is a closed convex cone in $E^{*}$. As in the proof of Lemma 4.1, we may assume that there exists a sunny nonexpasnsive retraction $R$ of $E$ onto $H$. In this case, we have $R(E)=F(R)=H$. Define a mapping $\hat{R}: E \rightarrow E$ by $\hat{R}(x)=$ $-R(-x)$ for all $x \in E$. For any $x \in E$, we have $R(-x) \in H$ and $\hat{R} x \in-H$. When $x \in-H$, we have $-x \in F(R)$ and $\hat{R} x=-R(-x)=-(-x)=x$. Then we have that $\hat{R}(E)=F(\hat{R})=-H$. For any $x, y \in E$,

$$
\begin{aligned}
\|\hat{R} x-\hat{R} y\| & =\|-R(-x)+R(-y)\| \\
& \leq\|x-y\| .
\end{aligned}
$$

Then $\hat{R}$ is a nonexpansive retraction of $E$ onto $-H$. As in the proof of Lemma 4.1, $R$ (resp. $\hat{R}$ ) maps any point $x \notin H$ (resp. $x \notin-H$ ) to the boundary $(-H) \cap H=$ $M$. Then $\hat{R} \circ R$ is a nonexpansive retraction onto $(-H) \cap H=M$. Indeed, $\hat{R} \circ R$ is a nonexpansive mapping. So we shall show that it is a retraction of $E$ onto $M$. If $x \in M$, then $\hat{R} \circ R x=x \in M$. If $x \in H \backslash M$, then $R x=x \in H \backslash M$ and $\hat{R} \circ R x \in M$. If $x \in(-H) \backslash M$, then $R x \in M$ and $\hat{R} \circ R x \in M$. Then, we have that $F(\hat{R} \circ R)=\hat{R} \circ R(E)=M$.

From Theorem 3.5, JM is a closed convex cone in $E^{*}$. Since $M$ is a closed linear subspace of $E$, for any $x^{*} \in J$ and $\alpha \in \mathbb{R}$, we have $\alpha x^{*} \in J M$. Then $J M$ is a closed linear subspace in $E^{*}$.

When $J M$ is a closed linear subspace of $E^{*}$, there exists a norm one linear projection $P$ of $E$ onto $M$; see $[10,18]$. We define the new operator $Q: E \rightarrow E$ such that

$$
Q x= \begin{cases}P x & \text { if } x \notin H, \\ x & \text { if } x \in H .\end{cases}
$$

$Q$ is a nonlinear retraction of $E$ onto $H$. We shall show that $Q$ is nonexpansive. When $x, y \in H$ or $x, y \in E \backslash H$, we have $\|Q x-Q y\| \leq\|x-y\|$, obviously. When $x \in H$ and $y \in E \backslash H$, let $z$ be an element of the segument $[x, y]$ such that $z \in M$. We have that

$$
\begin{aligned}
\|Q x-Q y\| & =\|x-P y\| \leq\|x-z\|+\|z-P y\| \\
& =\|x-z\|+\|P z-P y\| \leq\|x-z\|+\|z-y\| \\
& =\|x-y\| .
\end{aligned}
$$

Then, $Q$ is a nonexpansive retraction of $E$ onto $H$. So, $H$ is a nonexpansive retract of $E$.

To prove Theorem 4.1, we need more lemmas; 
Lemma 4.3. Let $E$ be a Banach space and let $K$ be a closed convex cone in $E$ such that for some $z^{*} \in E^{*} \backslash\{0\}$

$$
K \supset M:=\left\{x \in E:\left\langle x, z^{*}\right\rangle=0\right\} .
$$

Then $K$ is one of the following four;

(1) the closed hyperplane $M$;

(2) the closed half-space $H_{+}=\left\{x \in E:\left\langle x, z^{*}\right\rangle \geq 0\right\}$;

(3) the closed half-sapce $H_{-}=\left\{x \in E:\left\langle x, z^{*}\right\rangle \leq 0\right\}$;

(4) the whole space E.

Proof. Suppose that $K$ contains an element $\xi \in E$ such that $\left\langle\xi, z^{*}\right\rangle=a>0$. For any $y \in E$ such that $0<\left\langle y, z^{*}\right\rangle<a$, we define $y_{\alpha}$ as follows:

$$
y_{\alpha}=\alpha(y-\xi)+\xi, \quad \alpha \geq 0 .
$$

When $\alpha=0$, we have $\left\langle y_{\alpha}, z^{*}\right\rangle=a>0$. As $\alpha \rightarrow \infty,\left\langle y_{\alpha}, z^{*}\right\rangle$ decreases strictly and continuously. Furthermore, it tends to $-\infty$. Then there exists $\alpha_{0}>0$ such that $\left\langle y_{\alpha_{0}}, z^{*}\right\rangle=0$. This means that there exist $x \in M$ and $\alpha>0$ such that

$$
x=\alpha(y-\xi)+\xi .
$$

So, we have

$$
y=\frac{1}{\alpha} x+\left(1-\frac{1}{\alpha}\right) \xi .
$$

We can show $1<\alpha$. In fact, if $\alpha=1$, then $\left\langle y, z^{*}\right\rangle=\left\langle x, z^{*}\right\rangle=0$. This is a contradiction. If $0<\alpha<1$, then $\left\langle y, z^{*}\right\rangle=\frac{1}{\alpha}\left\langle x, z^{*}\right\rangle+\left(1-\frac{1}{\alpha}\right)\left\langle\xi, z^{*}\right\rangle=$ $\left(1-\frac{1}{\alpha}\right) a<0$. This is a contradiction. So, we have $1<\alpha$.

Then $y$ is an element of the convex hull of $M \cup\{\xi\}$. So, we have

$$
K \supset\left\{x \in E: 0 \leq\left\langle x, z^{*}\right\rangle<a\right\} .
$$

Since $K$ is a closed convex cone, we have $K \supset H_{+}$.

Similarly, when $K$ contains an element $\zeta$ such that $\left\langle\zeta, z^{*}\right\rangle<0$, we have $K \supset$ $H_{-}$. Then if $K \neq M$, then $K \supset H_{+}$or $K \supset H_{-}$. The proof is completed.

Lemma 4.4. Let $E$ be a Banach space and let $M$ be a hyperplane in $E$ such that for some $z^{*} \in E^{*} \backslash\{0\}$,

$$
M=\left\{x \in E:\left\langle x, z^{*}\right\rangle=0\right\} .
$$

Then $M^{\perp}=\overline{\operatorname{span}}\left\{z^{*}\right\}$, where $\overline{\operatorname{span}}\left\{z^{*}\right\}=\left\{x^{*} \in E^{*}: x^{*}=\alpha z^{*}, \alpha \in \mathbb{R}\right\}$. 
Proof. It is clear that $M^{\perp} \supset \overline{\operatorname{span}}\left\{z^{*}\right\}$. It is sufficient to show that there exists a unique non-zero element in $E^{*}$ up to a scalar multiple, such that it vanishes in $M$.

Since $M$ is a hyperplane, for $x_{0} \in E \backslash M$, we have

$$
E=\overline{\operatorname{span}}\left\{M \cup\left\{x_{0}\right\}\right\},
$$

where $\overline{\operatorname{span}} A$ is a closed linear span generated by $A$. For any $x \in \operatorname{span}\left\{M \cup\left\{x_{0}\right\}\right\}$, we can say $x=\alpha x_{0}+m$, where $\alpha$ and $m$ are some real value and some element of $M$, respectively. Then, we have taht for any $x \in \operatorname{span}\left\{M \cup\left\{x_{0}\right\}\right\},\left\langle x, z^{*}\right\rangle=$ $\alpha\left\langle x_{0}, z^{*}\right\rangle$ and $\left\langle x_{0}, z^{*}\right\rangle \neq 0$. If $w^{*} \in M^{\perp}$, then for any $x \in \operatorname{span}\left\{M \cup\left\{x_{0}\right\}\right\}$, $\left\langle x, w^{*}\right\rangle=\alpha\left\langle x_{0}, w^{*}\right\rangle$. This means that $\left\langle x, w^{*}\right\rangle=\frac{\left\langle x_{0}, w^{*}\right\rangle}{\left\langle x_{0}, z^{*}\right\rangle}\left\langle x, z^{*}\right\rangle$. Since $w^{*}$ and $z^{*}$ are continuous, we have $\left\langle x, w^{*}\right\rangle=\frac{\left\langle x_{0}, w^{*}\right\rangle}{\left\langle x_{0}, z^{*}\right\rangle}\left\langle x, z^{*}\right\rangle$ for any $x \in E$. So, we have $w^{*}=\frac{\left\langle x_{0}, w^{*}\right\rangle}{\left\langle x_{0}, z^{*}\right\rangle} z^{*}$ and hence $w^{*} \in\left\{x^{*} \in E^{*}: x^{*}=\alpha z^{*}, \alpha \in \mathbb{R}\right\}$.

Let $E$ be a Banach space and let $Y_{1}, Y_{2} \subset E$ be closed linear subspaces. If $Y_{1} \cap Y_{2}=\{0\}$ and for any $x \in E$ there exists a unique pair $y_{1} \in Y_{1}, y_{2} \in Y_{2}$ such that

$$
x=y_{1}+y_{2},
$$

then, we represent the space $E$ as

$$
E=Y_{1} \oplus Y_{2}
$$

Lemma 4.5. Let $E$ be a strictly convex, reflexive and smooth Banach space and let $Y^{*}$ be a closed linear subspace of the dual space $E^{*}$ of $E$ such that for any $y_{1}, y_{2} \in J^{-1} Y^{*}, y_{1}+y_{2} \in J^{-1} Y^{*}$. Then, $J^{-1} Y^{*}$ is a closed linear subspace of $E$ and the sunny generalized nonexpansive retraction $R_{Y^{*}}=J^{-1} \Pi_{Y^{*}} J$ of $E$ onto $J^{-1} Y^{*}$, where $\Pi_{Y^{*}}$ is the generalized projection of $E^{*}$ onto $Y^{*}$, is a norm one linear projection of $E$ onto $J^{-1} Y^{*}$. Further, the following holds:

$$
E=J^{-1} Y^{*} \oplus Y_{\perp}^{*} \text {. }
$$

Proof. By the assumption, for any $y_{1}, y_{2} \in J^{-1} Y^{*}$, we have $y_{1}+y_{2} \in J^{-1} Y^{*}$. Further, for $y \in J^{-1} Y^{*}$ and $\alpha \in \mathbb{R}$, we have from $J(\alpha y)=\alpha J y \in Y^{*}$ that $\alpha y \in J^{-1} Y^{*}$. So, $J^{-1} Y^{*}$ is a linear subspace of $E$. Since $J$ is norm to weak continuous and $Y^{*}$ is weakly closed subset in $E^{*}, J^{-1} Y^{*}$ is closed. Therefore, $J^{-1} Y^{*}$ is a closed linear subspace of $E$. For any $x, y \in E$, from Theorem 3.1, we have $R_{Y^{*}} x, R_{Y^{*}} y \in J^{-1} Y^{*}$. Since $J^{-1} Y^{*}$ is a linear subspace of $E$, we have $R_{Y^{*}} x+R_{Y^{*}} y \in J^{-1} Y^{*}$. Since $Y^{*}$ is a closed linear subspace of $E^{*}$, from Lemma 2.3, for any $x \in E$, an element $y \in J^{-1} Y^{*}$ satisfies $y=R_{Y^{*}} x$ if and only if

$$
\left\langle x-y, m^{*}\right\rangle=0, \quad \forall m^{*} \in Y^{*} .
$$


For $x \in E$ and $\alpha \in \mathbb{R}$, let $y=R_{Y^{*}} x$. We have that

$$
\left\langle\alpha x-\alpha y, m^{*}\right\rangle=0, \quad \forall m^{*} \in Y^{*} .
$$

Since $\alpha y \in J^{-1} Y^{*}$, we have that

$$
\alpha y=R_{Y^{*}}(\alpha x) .
$$

For $x_{1}, x_{2} \in E$, let $y_{1}=R_{Y^{*}} x_{1}$ and $y_{2}=R_{Y^{*}} x_{2}$. Then, we have that

$$
\left\langle x_{1}+x_{2}-\left(y_{1}+y_{2}\right), m^{*}\right\rangle=\left\langle x_{1}-y_{1}, m^{*}\right\rangle+\left\langle x_{2}-y_{2}, m^{*}\right\rangle=0, \quad \forall m^{*} \in Y^{*} .
$$

Since $y_{1}+y_{2} \in J^{-1} Y^{*}$, we obtain that

$$
R_{Y^{*}}\left(x_{1}+x_{2}\right)=y_{1}+y_{2}=R_{Y^{*}} x_{1}+R_{Y^{*}} x_{2} .
$$

So, the retraction $R_{Y^{*}}$ is linear. Since $\phi\left(R_{Y^{*}} x, m\right) \leq \phi(x, m)$ for any $x \in E$ and $m \in J^{-1} Y^{*}$, putting $m=0 \in J^{-1} Y^{*}$, we have that

$$
\left\|R_{Y^{*}} x\right\| \leq\|x\| .
$$

Then, $R_{Y^{*}}$ is a norm one linear projection of $E$ onto $J^{-1} Y^{*}$.

From this, we have that

$$
E=J^{-1} Y^{*} \oplus R_{Y^{*}}^{-1} 0
$$

where $R_{Y^{*}}^{-1} 0=\left\{x \in E: R_{Y^{*}} x=0\right\}$. It is sufficient to show that $R_{Y^{*}}^{-1} 0=Y_{\perp}^{*}$. From (4.3), we have that

$$
x \in R_{Y^{*}}^{-1} 0 \Leftrightarrow\left\langle x, m^{*}\right\rangle=0, \quad \forall m^{*} \in Y^{*} .
$$

This means that

$$
R_{Y^{*}}^{-1} 0=Y_{\perp}^{*}
$$

Proof of Theorem 4.1. Let $H$ be a closed half-space of $E$ such that for some $z^{*} \in E^{*} \backslash\{0\}$,

$$
H=\left\{x \in E:\left\langle x, z^{*}\right\rangle \leq 0\right\} .
$$

When $J H$ is a closed half-space in $E^{*}, J H$ is a closed convex cone in $E^{*}$. So, from Lemma 4.1, $H$ is a nonexpansive retract of $E$. It is sufficient to show that if $H$ is a nonexpansive retract of $E$, then $J H$ is a closed half-space in $E^{*}$.

Assume $H$ is a nonexpansive retract of $E$. From Lemma 4.1, $J H$ is closed convex cone in $E^{*}$. From Lemma 4.2, $J M$ is a closed linear subspace in $E^{*}$, where $M=\left\{x \in E:\left\langle x, z^{*}\right\rangle=0\right\}$. Since $M \subset E=E^{* *}$ and $J_{*}^{-1} M=J M$ is a closed linear subspace in $E^{*}$, from Lemma 4.5 , we have that

$$
E^{*}=J_{*}^{-1} M \oplus M^{\perp},
$$


where $M^{\perp}=\left\{x^{*} \in E^{*}:\left\langle x^{*}, m\right\rangle=0 \quad \forall m \in M\right\}$. Then, from Lemma 4.4, we have that

$$
E^{*}=J M \oplus \overline{\operatorname{span}}\left\{z^{*}\right\} .
$$

This means that the co-dimension of the closed linear subspace $J M$ in $E^{*}$ is one. Then, $J M$ is a closed hyperplane in $E^{*}$.

Since the closed conve cone $J H$ contains the hyperplane $J M$, the duality mapping $J$ is bijective and both sets $H \backslash M$ and $E \backslash H$ are nonempty, from Lemma 4.3, we obtain that $J H$ is a closed half-space in $E^{*}$. This completes the proof.

From this theorem, we obtain the following corollary.

Corollary 4.1. Let $E$ be a strictly convex, smooth and reflexive Banach space and let $H$ be a closed half-space of $E$ such that for some $z^{*} \in E^{*}$

$$
H=\left\{x \in E:\left\langle x, z^{*}\right\rangle \leq 0\right\} .
$$

Then, $J H$ is a closed convex cone in $E^{*}$ if and only if $J H$ is a closed half-space in $E^{*}$.

Remark 4.1. In a Hilbert space, the normalized duality mapping $J$ is the identity mapping. The image of a closed convex cone by $J$ is always a closed convex cone and the image of a closed half-space by $J$ is always a closed half-space. In this case, any closed convex cone is a nonexpansive retract; see [36].

Remark 4.2. Let $E$ be a strictly convex, smooth and reflexive Banach space, let $z \in E$ and let $M^{*}=\{\overline{\operatorname{span}}\{z\}\}^{\perp}$. When $P_{\overline{\operatorname{span}}\{z\}}$ is linear, $R_{M^{*}}$ is a norm one linear projection onto $J^{-1} M^{*}$; see $[10,18]$. Then $M^{*}$ is a closed hyperplane such that $J^{-1} M^{*}=J_{*} M^{*}$ is a closed linear subspace of $E$.

In $[13,14]$, Deutsch showed an equivalent condition for the metric projection $P_{\overline{\mathrm{span}}\{z\}}$ to be linear in $L^{p}$ spaces; see also $[6,16]$.

\section{ACKNOWLEDGMENT}

This work was supported by the Grant NSC 98-2115-M-110-001.

\section{REFERENCES}

1. Ya. I. Alber, Metric and generalized projection operators in Banach spaces: properties and applications, Theory and Applications of Nonlinear Operators of Accretive and Monotone Type, Dekker, New York, 1996, pp. 15-50. 
2. Ya. I. Alber, Generalized Projections, Decompositions and the Pythagorean-Type Theorem in Banach Spaces, Appl. Math. Lett., 11 (1998), 115-121.

3. Ya. I. Alber, James orthogonality and orthogonal decompositions of Banach spaces, J. Math. Anal. Appl., 312 (2005), 330-342.

4. G. Ascoli, Sugli spazi lineari metrici e le loro varieta lineari, Ann. Mat. Pura. Appl., 10 (1932), 33-81, 203-232.

5. B. Beauzamy, Projections contractantes dans les espaces de Banach, Bull. Sci. Math., 102 (1978), 43-47.

6. B. Beauzamy and B. Maurey, Points minimaux et ensembles optimaux dans les espaces de Banach, J. Functional Anal., 24 (1977), 107-139.

7. G. Birkhoff, Orthogonality in linear metric spaces, Duke Math. J., 1 (1935), 169-172.

8. N. Bourbaki, Espaces vectoriels topologiques, Ch. I-II, Hermann et Cie, Paris, 1952; Ch. III-V, Hermann et Cie, Paris, 1955.

9. R. E. Bruck, Nonexpansive projections on subsets of Banach spaces, Paciffic J. Math., 47 (1973), 341-355.

10. B. Calvert, Convergence sets in reflexive Banach spaces, Proc. Amer. Math. Soc., 47 (1975), 423-428.

11. I. Cioranescu, Geometry of Banach spaces, Duality Mappings and Nonlinear Problems, Kluwer Academic Publishers, Dordecht, 1990.

12. W. Davis and P. Enflo, Contractive projections on $l^{p}$-spaces, London Math. Soc. Lecture Note Series, 137 (1989), 162-184.

13. F. Deutsch, Linear selections for the metric projection, J. Funct. Anal., 49 (1982), 269-292.

14. F. Deutsch, A survey of metric selections, Contemp. Math., 18 (1983), 49-71.

15. P. Enflo, Contractive projections onto subsets of $L^{1}(0,1)$, London Math. Soc. Lecture Notes Series, 137 (1989), 151-161.

16. P. Enflo, Contractive projections onto subsets of $L^{p}$-spaces, Lecture Notes in Pure and Applied Mathematics, 136 (1992), 79-94.

17. M. J. Fabian, P. Habala, P. Hajek and J. Pelant, Functional analysis and infinitedimensional geometry, Springer Verlag, 2001.

18. T. Honda and W. Takahashi, Nonlinear projections and generalized conditional expectations in Banach spaces, Taiwanese J. Math., to appear.

19. T. Honda and W. Takahashi, Norm One Projections and Generalized Conditional Expectations, Scientiae Mathematicae Japonicae, 69 (2009), 303-313.

20. H. Honda, T. Ibaraki and W. Takahashi, Duality theorems and convergence theorems for nonlinear mappings in Banach spaces and applications, Int. J. Math. Stat., 6 (2010), 46-64 
21. H. Hudzik, Y. Wang and R. Sha, Orthogonally complemented subspaces in Banach spaces, Nonlinear Analysis, Mumer. Funct. Anal. Optim., 29 (2008), 779-790.

22. T. Ibaraki and W. Takahashi, A new projection and convergence theorems for the projections in Banach spaces, J. Approx. Theory, 149 (2007), 1-14.

23. T. Ibaraki and W. Takahashi, Fixed point theorems for nonlinear mappings of nonexpansive type in Banach spaces, J. Nonlinear Convex Anal., 10 (2009), 21-32.

24. S. Itoh and W. Takahashi, The common fixed point theory of singlevalued mappings and multivalued mappings, Pacific J. Math., 79 (1978), 493-508.

25. R. C. James, Orthogonality in normed linear spaces, Duke Math. J., 12 (1945), 291-302.

26. R. C. James, Orthogonality and linear functionals in normed linear spaces, Trans. Amer. Math. Soc., 61 (1947), 265-292.

27. R. C. James, Inner products in normed linear spaces, Bull. Amer. Math. Soc., 53 (1947), 559-566.

28. S. Kamimura and W. Takahashi, Strong convergence of a proximal-type algorithm in a Banach space, SIAM J. Optim., 13 (2002), 938-945.

29. F. Kohsaka and W. Takahashi, Generalized nonexpansive retractions and a proximaltype algorithm in Banach spaces, J. Nonlinear Convex Anal., 8 (2007), 197-209.

30. E. Kopecká and S. Reich, Nonexpansive retracts in Banach spaces, Banach Center Publications, 77 (2007), 161-174.

31. R. E. Megginson, An introduction to Banach space theory, Springer-Verlag, BerlinHeidelberg-New York, 1998.

32. R. R. Phelps, Convex sets and nearest points, Proc. Amer. Math. Soc., 8 (1957), 790-797.

33. B. Randrianantoanina, Norm one projections in Banach spaces, Taiwanese J. Math., 5 (2001), 35-95.

34. S. Reich, Extension problems for accretive sets in Banach spaces, J. Funct. Anal., 26 (1977), 378-395.

35. I. Singer, Best approximation in normed linear spaces by elements of linear subspaces, Springer-Verlag, Berlin-Heidelberg-New York, 1970.

36. W. Takahashi, Nonlinear Functional Analysis-Fixed Point Theory and Its Applications, Yokohama Publishers, 2000.

37. W. Takahashi, Convex Analysis and Approximation of Fixed Points, Yokohama Publishers, 2000, (in Japanese).

38. W. Takahashi and J.-C. Yao, Nonlinear operators of monotone type and convergence theorems with equilibrium problems in Banach spaces, Taiwanese J. Math., to appear.

39. R. Wittmann, Hopfs ergodic theorem for nonlinear operators, Mathematische Annalen, 289 (1991), 239-253. 
Takashi Honda

Department of Applied Mathematics,

National Sun Yat-sen University,

Kaohsiung 80424, Taiwan

E-mail: honda@mail.math.nsysu.edu.jp

\section{Wataru Takahashi}

Department of Applied Mathematics, National Sun Yat-sen University,

Kaohsiung 80424, Taiwan

E-mail: wataru@is.titech.ac.jp

Jen-Chih Yao

Department of Applied Mathematics, National Sun Yat-sen University,

Kaohsiung 80424, Taiwan

E-mail: yaojc@math.nsysu.edu.tw 